\title{
Additivity in prism adaptation as manifested in intermanual and interocular transfer
}

\author{
BENJAMIN WALLACE \\ Cleveland State University, Cleveland, Ohio 44115 \\ and \\ GORDON M. REDDING \\ Illinois State University, Normal, Illinois 61661
}

\begin{abstract}
Level of adaptation was assessed in both exposed and unexposed eye and/or hand for visual shift (VS), proprioceptive shift (PS), and the eye-hand coordination, negative after effect (NA) measure of both visual and proprioceptive change, following 15-min and 20-diopter base-right displacement viewing of the active hand, under conditions of unconstrained head movement and terminal exposure feedback. Transfer was complete for the VS test, and significant, but incomplete for the PS and NA tests. For both exposed and unexposed eye/hand situations, level of adaptation was greater for the NA than for the PS test, which in turn showed greater adaptation than the VS test. Additivity was virtually perfect for the unexposed eye/hand (VS+PS = NA), but underadditivity appeared for the exposed eye/hand (VS+PS < NA). This underadditivity was approximately equal in magnitude to the amount that transfer on the NA test was less than on the PS test, suggesting that underadditivity was due to a nontransferable, assimilated corrective response in the NA test with the exposed eye/hand. Possible explanations for intermanual transfer are discussed.
\end{abstract}

It is now reasonably clear that in an eye-hand coordination task involving an exposure to a prismatically transformed environment, adaptation to such a situation can be described in terms of an additive relationship between various components (Hay \& Pick, 1966; McLaughlin \& Webster, 1967; Redding, 1978; Redding \& Wallace, 1976, 1978; Wallace, 1977; Wilkinson, 1971). Specifically, when individuals adapt to prismatic displacement, such adaptation appears to involve the entire control loop that regulates their interaction with the environment. Such adaptation can generally be represented by two components of the control loop: one responsible for felt limb location/orientation, and one responsible for visual location/orientation of a perceived stimulus.

In displacement adaptation, the felt-limb-position component is assessed in the absence of a perceived visual stimulus. Thus, individuals are simply required to position an unseen limb until they believe it to be in the straight-ahead position, before and after an exposure to prismatic displacement. Since a visual stimulus is not present, errors in felt-limb localization are believed to be the result of a change in significance of proprioceptive input to the control

The authors acknowledge the assistance of Johann Tolnai in data collection. Reprint requests should be sent to Benjamin Wallace, Department of Psychology, Cleveland State University, Cleveland, Ohio 44115. loop. This preexposure-postexposure difference in straight-ahead localization accuracy is commonly referred to as the proprioceptive shift (PS).

The visual component is generally assessed as a localization difference between the preexposure and the postexposure location of a visual stimulus. This measure, or difference score, is commonly referred to as the visual shift (VS). Total adaptation to a prismatic displacement in an eye-hand coordination task (referred to as a negative aftereffect, NA) is usually the sum of visual and proprioceptive inputs to the control loop.

Several investigations (Choe \& Welch, 1974; McLaughlin \& Bower, 1965; Wallace, 1978; Melamed, Beckett, \& Hill, Note 1) have shown that in some situations, the proprioceptive component is amenable to intermanual transfer. In other words, the recalibrated proprioceptive input seems to influence control of the unexposed limb. Estimates of PS transfer vary, depending upon exposure conditions (Welch, 1978). However, the situation which has been shown to maximize the magnitude of intermanual transfer is when a subject is permitted some head movement during prism exposure while performing localization actions in a terminal reaching mode where the consequences of a localization action are perceived only during the final phase of the movement (Wallace, 1978).

There is also evidence to indicate that visual inputs from the two eyes contribute equally to the 
control loop (Ebenholtz, 1970). Several studies (Crawshaw \& Craske, 1976; Foley \& Miyanshi, 1969) have found interocular transfer between the exposed and unexposed eyes in a prism displacement situation. However, unlike intermanual transfer, interocular transfer appears to be complete $(100 \%)$.

The transferability of visual and proprioceptive adaptation offers the opportunity for a further test of the additivity hypothesis. If the eyes and limbs provide visual and proprioceptive input to a common control loop, then the total transferable adaptation (NA) should be described in an additive fashion, such that transferable VS and transferable PS combine in a manner similar to that observed for the exposed eye and exposed limb. The purpose of the present experiment was to test this hypothesis. For each subject, VS, PS, and NA were measured for both the exposed and unexposed eye and/or hand. Unconstrained head movement and terminal exposure conditions were used to maximize intermanual transfer, and, to control for possible biases due to eye and hand dominance, different groups received different exposure combinations of dominant and nondominant, eye and hand.

\section{METHOD}

\section{Subjects}

Thirty-two, right-hand-dominant and right-eye-dominant individuals (18 females and 14 males) served as subjects. Hand dominance and eye dominance were determined by the subjects' responses to questions (e.g., With which hand do you write? With which eye would you observe through a telescope? etc.). All subjects were volunteers from the introductory psychology classes at Cleveland State University. Also, all subjects had normal, $20 / 20$ uncorrected visual acuity and normal motor dexterity.

\begin{abstract}
Apparatus
The apparatus was similar to that employed by Wallace (1978). Specifically, this structure consisted of a two-layer, rectangular, wooden, box-like frame $(77 \mathrm{~cm}$ high, $62 \mathrm{~cm}$ wide, and $62 \mathrm{~cm}$ deep) open on the side facing the subject. When a subject placed his/her arm within the structure, on the lower layer, the arm was not visible. In this area, during test, arm position could be noted by the experimenter by determining its location along a $180^{\circ}$ arc, calibrated in $1^{\circ}$ increments. During exposure, placement of the subject's arm upon the upper layer permitted the individual to perceive arm movement only at the terminus of a response action. Observation of arm movement throughout most of the exposure period was prevented by a plywood cover, located $12 \mathrm{~cm}$ directly above the second layer of the apparatus. During exposure, all visible surfaces were homogeneous in appearance, except for a target $(.2 \times 8 \mathrm{~cm})$ positioned in the physical straight-ahead location. Attached to the apparatus was a Marietta Instruments combination head- and chinrest. The chin portion of the head constraint could be loosened to permit head swivel during unconstrained head movement.

\section{Design}

Eight subjects were randomly assigned to each of the four conditions determined by combinations of dominant and nondominant, eye and hand during exposure. All subjects received three localization tests (VS, PS, and NA), and both exposed and and unexposed eye and/or hand were tested for each subject.
\end{abstract}

Order of localization tests and transfer tests (i.e., exposed/ unexposed eye/hand) was randomized. Such randomization was deemed necessary to equate for possible spontaneous decay in the various localization tasks.

\section{Procedure}

The procedure was the same as that employed by Wallace (1978). Specifically, each subject was tested individually by being led, blindfolded, to the experimental room and seated in a chair before the apparatus. The blindfold consisted of Risley prisms set to 0 diopters and mounted in welder's goggles, with vision occluded by a thick flap over each eyepiece. The subject's head was then positioned in the head- and chinrest. The experimenter then asked the subject to perform each of the three localization tests, in a random order.

The PS test required the subjects to place dominant or nondominant hands (random starting order) on the lower layer of the apparatus, and to point to the position in space believed to be straight ahead of their noses. This task was accomplished with vision occluded. During preexposure, baseline determination, straight-ahead localization was performed 10 times with each hand, randomly alternating hands across the total 20 pointing trials.

The NA test was similar, except the subject was not blindfolded, but pointed to a visible, vertical target $(.2 \times 8 \mathrm{~cm})$ located in the physical straight-ahead position. During this test, the subject viewed the target with no visual displacement (i.e., 0-diopter prism setting), but pointing accuracy was not known to the subject since the hand was on the lower layer of the apparatus. Only two combinations of eye and hand were used for the NA test which corresponded to the exposed eye/hand combination and the unexposed eye/hand combination for a given treatment group. Ten measures were taken for each eye/hand combination used. Order of localization actions was random.

The VS test required no arm movement. Instead, the experimenter introduced a moving, visible, vertical target $(.2 \times 8 \mathrm{~cm})$ on the upper layer of the apparatus. When the experimenter moved this target in a lateral fashion across the subject's visual field, the subjects simply indicated when the target appeared to be straight ahead of their noses. A total of 20 trials were given on this test. Half of the trials required observation of the target with the dominant eye, and half with the nondominant eye. For the 10 trials with a given eye, 5 started with the target randomly positioned in the left half of the subject's visual field and 5 started with the target randomly positioned in the right half of the visual field. Order of the exposed eye starting position was random across the 20 trials. The prisms were set at 0 diopters for the VS test, as was true for all test situations in the experiment.

Following establishment of VS, PS, and NA baselines, a subject was randomly assigned to one of the four conditions of dominance for the exposed eye and hand. During a 15-min terminal exposure to 20-diopter, base-right prisms, the subject was asked to point in a sagittal, ballistic fashion to a visible target $(.2 \times$ $8 \mathrm{~cm}$ ). Pointing actions were monitored and paced such that each back-and-forth movement required approximately $3 \mathrm{sec}$ for completion. Also, during this exposure, the chinrest of the headand-chin constraint was loosened to permit head swivel and movement.

Immediately after the exposure session the chinrest of the headand-chin constraint was tightened and positioned as it was in the preexposure portion of the experiment. The localization tests were then repeated. Level of adaptation was specified as the signed difference between mean pretest and posttest for each localization measure. For the NA and PS tests, a change in the direction opposite prismatic displacement was taken to be positive and adaptive, while for the VS test, a change in the direction of prismatic displacement was scored as positive and adaptive (Harris, 1974; Welch, 1974). All subjects in the study showed adaptive shifts on all three localization tests, with the exception 
of one subject, in the dominant-hand/dominant-eye exposure condition, who exhibited a maladaptive and nonsignificant proprioceptive shift. This individual was replaced in the experiment.

\section{RESULTS}

A preliminary analysis of the data indicated the absence of a trials effect for the VS, PS, and NA localization tests. Consequently, subsequent analyses were restricted to mean scores for each subject on each of the three localization tests. An analysis of variance was performed with the between-group factors, hand (dominant or nondominant) and eye (dominant and nondominant), and the within-group factors, transfer test (exposed or unexposed eye and/ or hand), and localization measure (VS, PS, or NA).

There were no significant between-group main effects. All $\mathrm{F}$ values were less than or approximated 1.00. There was, however, a tendency for level of adaptation to be greater when the dominance relationship between hand and eye was different (i.e., for the groups receiving exposure with the dominant eye and nondominant hand or dominant hand and nondominant eye) $[F(1,28)=3.41, p<.10]$, and this effect was restricted to the PS and NA tests $[F(2,56)=2.49, \mathrm{p}<.10]$.

The only other significant sources of variance were main effects for transfer tests $[F(1,28)=43.79$, $\mathrm{p}<.001]$ and localization measures $[\mathrm{F}(2,56)=82.95$, $\mathrm{p}<.001]$ and the interaction of these two variables $[F(2,56)=22.28, p<.001]$. Means for these effects are reported in Table 1. As can be seen, adaptation was greatest for the NA test, intermediate for the PS test, and least for the VS test, but this effect was greatest for the exposed eye/hand, and the difference between transfer tests was restricted entirely to the NA and PS tests. Subsequent comparisons demonstrated statistical reliability $(p<.001)$ for the pattern VS < PS < NA for both exposed and unexposed eye/hand test conditions.

Also shown in Table 1 is the percent transfer for

Table 1

Mean Level of Adaptation (Degrees) and Confidence Limits (95\%) for Localization Tests of Visual Shift (VS), Proprioceptive Shift (PS), and Negative Aftereffect (NA) as a Function of Testing with the Exposed or Unexposed Eye and/or Hand

\begin{tabular}{llllll}
\hline & \multicolumn{5}{c}{ Localization Test } \\
\cline { 2 - 6 } \multicolumn{1}{c}{ Transfer Test } & VS & PS & NA & VS+PS & D \\
\hline Exposed Eye/Hand & 1.6 & 3.2 & 5.2 & 4.8 & .4 \\
Unexposed Eye/Hand & $(.3)$ & $(.6)$ & $(.7)$ & & \\
& 1.6 & 2.2 & 3.9 & 3.8 & .1 \\
Transfer & $(.3)$ & $(.4)$ & $(.6)$ & & \\
\hline
\end{tabular}

Note-Also shown is the difference (D) between NA and the sum VS+PS for each transfer test and the percent transfer for each localization test. each of the three localization tests. The VS test revealed complete interocular transfer $(100 \%)$, while intermanual transfer on the PS test was smaller in magnitude $(69 \%)[\mathrm{t}(31)=4.12, \mathrm{p}<.002]$, but not statistically different from intermanual transfer as measured by the NA test $(75 \%)$ [ $\mathrm{t}(31)=1.94]$.

Tests for additivity, within each transfer test, were performed by comparing the NA score with the sum of VS and PS scores for each subject. As can be seen in Table 1, additivity was virtually perfect for the unexposed eye/hand $[\mathrm{t}(31)=0.84]$, but significant underadditivity occurred for the exposed eye/hand $[\mathrm{t}(31)=2.37, \mathrm{p}<.05]$. The sum VS + PS was less than the NA by $.4^{\circ}$.

\section{DISCUSSION}

The results for the unexposed eye/hand test condition provide still further support for the additivity hypothesis. Under a variety of exposure and test conditions (e.g., Redding \& Wallace, 1976, 1978), and now in transfer to the unexposed eye/hand, VS and PS components have been shown to combine in a simple, linear fashion to produce the total adjustment in the complete perceptual-motor control loop. Moreover, the deviation from additivity for the exposed eye/hand is easily explained. Previous studies (e.g., Templeton, Howard \& Wilkinson, 1974; Welch, Choe, \& Heinrich, 1974; Beckett, Melamed, \& Halay, Note 2) have also found a failure of the sum VS + PS to reach the NA value, and Welch $(1974,1978)$ has proposed that such underadditivity may be due to an "assimilated corrective response" component in the NA test. When the exposure period involves pointing at targets, the subject may quickly acquire a "rule" for correct pointing, which, with sufficient practice, can become automatic and unconscious and therefore persist for a time after exposure. Since this assimilated corrective response involves a learned eye-hand coordination with the exposed eye/hand, it should contribute to performance on the NA test, but not to either the PS or VS test. This "extra," assimilated corrective effect for the NA test would produce the observed underadditivity. However, it seems reasonable that such a response habit should be specific to the exposed eye/hand, and should generalize little, if at all, to the unexposed eye/hand. Therefore, the absence of this third component should lead to the observed additivity with the unexposed eye/hand. This interpretation of the present data is supported by the fact that the amount of nontransferable NA is more than nontransferable PS by approximately the amount of underadditivity shown for the exposed eye/hand, i.e.,.$^{\circ}$.

Removal of the assimilated-corrective-response contribution from the NA, exposed eye/hand score increases the estimated NA transfer $(80 \%)$, but, in 
absolute terms, still leaves about $1^{\circ}$ of adaptation which does not transfer in either the PS or NA test. Since transfer was obviously complete for the VS test, it seems reasonable to identify the nontransferable fraction with the PS test, but the nature of this PS effect is problematical. Intermanual transfer on the NA test is usually attributed to VS, which should appear regardless of which hand is tested (Welch, 1978). This is certainly a possibility here, particularly since interocular transfer was complete. However, interocular transfer cannot explain transfer on the PS test, where vision was precluded. Two previous studies have found such "direct" intermanual transfer, varying in magnitude from about $45 \%$ (Choe \& Welch, 1974) to $100 \%$ (McLaughlin \& Bower, 1965). A possible explanation of such transfer is that pointing straight-ahead is influenced by both felt limb position and by a "response tendency for pointing to an imagined (visualized) position in space"' (Welch, 1978, p. 95). Presumably, only the latter component transfers in the PS test. However, it seems reasonable to expect that any shift in visualized position should correspond to a change in the actual visual position, and the transferred components should be equal for VS and PS tests. In fact, if intermanual transfer is mediated solely, directly or indirectly, by vision, then adaptation for the unexposed eye/hand should be equal for all three tests, VS, PS, and NA. this was clearly not the case in the present experiment.

Alternatively, transfer on the PS test might be due to a change in felt head position. As a consequence of exposure, the head may come to feel turned (in the direction opposite the prism displacement), when in fact it is straight. If the head is taken as reference, such a change in felt head-trunk relationship would appear on the pointing tests (PS and NA), but not on the VS test. (If the trunk were taken as reference, a "correction" for felt head position would tend to reduce or eliminate any VS). This adaptation of the head-trunk system would transfer to the unexposed eye/hand while "true" proprioceptive adaptation of the limb (about $1^{\circ}$ ) would not.

Recalibration of felt head position has been suggested to explain intermanual transfer with visual targets (Hamilton, 1964) and with auditory targets (Cohen, 1974), but the only direct evidence for such a shift comes from Kohler (1964, p. 38), who reported that subjects eventually became unaware that their heads were turned in the direction of the displacement. Although the present data showing intermanual transfer and additivity may be interpreted by assuming a change in felt head position, they do not present any additional, direct evidence for such a change. Direct measures of felt head position, which unfortunately were not incorporated into the present study, must be added to future experiments investigating intermanual transfer before any definitive conclusion can be drawn.

\section{REFERENCE NOTES}

1. Melamed, L. E., Beckett, P. A., \& Hill, G. Individual differences in components of prism adaptation derived from the terminal exposure, distributed practice paradigm. Paper read at the meetings of the Midwestern Psychological Association, Chicago, May 1976.

2. Beckett, P. A., Melamed, L. E., \& Halay, M. Prism awareness, exposure duration, and the linear model in prism adaptation. Paper read at the meetings of the Midwestern Psychological Association, Chicago, May 1975.

\section{REFERENCES}

Chое, C. S., \& Welch, R. B. Variables affecting the intermanual transfer and decay of prism adaptation. Journal of Experimental Psychology, 1974, 102, 1076-1084.

COHEN, M. M. Changes in auditory localization following prismatic exposure under continuous and terminal visual feedback. Perceptual and Motor Skills, 1974, 38, 1202.

Crawshaw, M., \& Craske, B. Oculomotor adaptation to prisms: Complete transfer between eyes. British Journal of Psychology, 1976, 67, 475-478.

EnenHoltz, S. M. On the relation between interocular transfer of adaptation and Hering's law of equal innervation. Psychological Review, 1970, 77, 343-347.

Foley, J. E., \& MrYanshi, K. Interocular effects in prism adaptation. Science, 1969, 165, 311-312.

Hamilton, C. R. Intermanual transfer of adaptation to prisms. American Journal of Psychology, 1964, 77, 457-462.

Harris, C. S. Beware the straight-ahead shift: A nonperceptual change in experiments on adaptation to displaced vision. Perception, 1974, 3, 461-476.

HAY, J. C., \& PICK, H. L., JR. Visual and proprioceptive adaptation to optical displacement of the visual stimulus. Journal of Experimental Psychology, 1966, 71, 150-158.

KOHLER, I. The formation and transformation of the perceptual world. Psychological Issues, 1964, 3, 1-173.

Mclaughiin, S. C., \& Bower, J. L. Selective intermanual transfer of adaptive effects during adaptation to prisms. Psychonomic Science, 1965, 3, 69-70.

Mclaughlin, S. C., \& Webster, R. G. Changes in straightahead eye position during adaptation to wedge prisms. Perception \& Psychophysics, 1967, 2, 37-44.

ReDDING, G. M. Additivity in adaptation to optical tilt. Journal of Experimental Psychology: Human Perception and Performance, $1978,4,178-190$.

Redding, G. M., \& Wallace, B. Components of displacement adaptation in acquisition and decay as a function of hand and hall exposure. Perception \& Psychophysics, 1976, 20, 453-459.

RedDing, G. M., \& Wallace, B. Sources of "overadditivity" in prism adaptation. Perception \& Psychophysics, 1978, 24, 58-62.

Templeton, W. B., How Ard, I. P., \& Wilkinson, D. A. Additivity of components of prismatic adaptation. Perception \& Psychophysics, 1974, 15, 249-257.

WALLACE, B. Stability of Wilkinson's linear model of prism adaptation over time for various targets. Perception, 1977, 6, 145-151.

W ALLACE, B. Visuomotor coordination and intermanual transfer for a proprioceptive reaching task. Journal of Motor Behavior, $1978,10,139-147$.

WELCH, R. B. Research on adaptation to rearranged vision: 19661974. Perception, 1974, 3, 367-392.

Welch, R. B. Perceptual modification: Adapting to altered sensory environments. New York: Academic Press, 1978.

Welch, R. B., Choe, C. S., \& Heinrich, D. R. Evidence for a three-component model of prism adaptation. Journal of Experimental Psychology, 1974, 103, 700-705.

Wilkinson, D. A. Visual-motor control loop: A linear system? Journal of Experimental Psychology, 1971, 89, 250-257.

(Received for publication August 11, 1978; revision accepted December 4,1978 .) 